\title{
Stability against dynamic remodeling of an arterial tissue
}

\author{
I. M. Machyshyn - P. H. M. Bovendeerd • \\ A. A. F. van de Ven - P. M. J. Rongen • \\ F. N. van de Vosse
}

Received: 17 March 2008 / Accepted: 17 September 2009 / Published online: 4 October 2009

(C) The Author(s) 2009. This article is published with open access at Springerlink.com

\begin{abstract}
Geometry and structure of the arterial wall are maintained through continuous growth and remodeling (G\&R). To understand these processes, mathematical models have been proposed in which the outcome of G\&R depends on a mechanical stimulus through evolution equations. Rate parameters in these equations cannot be determined easily from experimental data. Assuming that the healthy artery is stable against remodeling, a physiologically acceptable range for the two rate parameters in the framework of an existing model of arterial G\&R is determined here. The model is explicitly evaluated for the example of a cylindrical blood vessel, both thick-walled and thin-walled. For the thin-walled vessel a criterion for stability against remodeling is derived by means of a linear stability approach, and is expressed in terms of the ratio of the rates of remodeling parameters. It is shown that this criterion is equivalent to the condition that the physiological healthy state of the artery can be reached, implying that if the healthy state exists then it is stable. Explicit numerical results are presented for a typical cerebral artery and an abdominal aorta.
\end{abstract}

Keywords Artery $\cdot$ Collagen $\cdot$ Homeostatic state $\cdot$ Remodeling $\cdot$ Stability

\section{Introduction}

Mechanoregulation mechanisms of arteries are activated in response to persistent changes of mechanical loading conditions of the arteries, initiating adaptation of the tissue. Long-term adaptation is realized through growth and remodeling $(\mathrm{G} \& \mathrm{R})$. Growth is defined as an increase in mass, and remodeling as a structural change of the tissue. $G \& R$ is striving to resume a homeostatic state of the artery, which, in a mechanical sense, can be related to either

I. M. Machyshyn · P. H. M. Bovendeerd · F. N. van de Vosse

Biomedical Engineering, Eindhoven University of Technology, Eindhoven, The Netherlands

I. M. Machyshyn

e-mail: i.machyshyn@tue.nl

A. A. F. van de Ven $(\varangle)$

Mathematics and Computing Science, Eindhoven University of Technology, Eindhoven, The Netherlands

e-mail: A.A.F.v.d.Ven@tue.nl

P. M. J. Rongen

Philips Medical Systems, Best, The Netherlands 
stress or strain criteria [1]. For instance, the arterial wall thickens because of persisting overpressure, leading to restoration of transmural stress [2, Chap. 13], whereas the inner diameter of an artery increases due to increased blood flow in order to restore normotensive shear stresses.

Apart from maintaining homeostasis of the healthy artery, G\&R is also involved in pathological formation of aneurysms. An aneurysm is a vascular disorder revealing itself by localized dilation or bulging of the artery. The most common locations of aneurysms are the abdominal aorta and the cerebral arteries. Aneurysms can rupture and then cause bleeding into the surrounding tissue. This is associated with serious medical complications and even death. Little is known about why aneurysms appear, how they grow, and what the criteria for their rupture are [3].

Mathematical models have been developed to describe arterial G\&R under normal and pathological circumstances. A framework for modeling growth, presented by Skalak et al. [4] and Rodriguez et al. [5] has been further developed and applied for the growth of arteries by, among others, Taber and Eggers [6], Taber and Humphrey [7], and Kuhl et al. [8]. An alternative growth model was introduced by Rachev et al. [9]. Other models have focused on cardiovascular-tissue remodeling, taking into account the microstructural composition of the tissue. Special attention has been paid to the description of remodeling of collagen, one of the most important load-bearing components. Driessen et al. [10-12] suggest models of collagen architecture and remodeling with application to arteries and heart valves. Gleason et al. [13] and Gleason and Humphrey [14] study arterial remodeling in response to altered mechanical loading, while Watton et al. [15] and Watton and Hill [16] describe collagen remodeling with application to abdominal aneurysms. Baek et al. [17, 18], Kroon and Holzapfel [19], Watton et al. [20], and Watton and Ventikos [21] model development of cerebral aneurysms. In all these studies, the G\&R outcome depends on the G\&R stimulus via evolution equations. Characteristic parameters for these evolution equations are the rate constants of G\&R. Unfortunately, these rates cannot be determined easily from experimental data.

The aim of this study is to provide a method to determine a physiologically acceptable range of values for the rate constants by means of a stability analysis. We use the natural assumption that the equilibrium state of the healthy artery is stable against remodeling, which means that, if the tissue does not experience any pathological changes, small changes of the mechanical properties of the tissue will cause remodeling of the tissue to a neighboring healthy state. This stability criterion narrows the range of candidate values for rate constants.

In our study we analyze the remodeling model of Watton et al. [15], which is one of the first structurally based models applied to aneurysm development. In [15] they assumed that the homeostatic state, which the artery always tends to maintain, is related to collagen stretch. At homeostasis collagen fibers are assumed to be stretched by a constant homeostatic stretch, $\lambda_{c}^{\text {hom }}$, independent of the position and the orientation of the fibers. This stretch is also independent of the actual state of the artery. If due to geometrical or structural changes the homeostatic state is violated and collagen stretch exceeds the homeostatic stretch, collagen acts to restore the equilibrium via two mechanisms. Firstly, by increasing the recruitment stretch of collagen, i.e., the tissue stretch at which initially undulated collagen fibers get straightened. In this way the onset of collagen recruitment shifts towards a larger tissue stretch, thus weakening the tissue. The restoration of the homeostatic collagen stretch is achieved through shifting a part of the load borne by collagen to elastin, another load-bearing component of the tissue. This mechanism is called remodeling of the recruitment stretch. Secondly, collagen can thicken so as to supplement the first mechanism in restoring homeostasis. Thickening is attributed to an increase in the density of collagen fibers, and is therefore called remodeling of collagen density. In contrast to the first mechanism, the second one stiffens the tissue. The remodeling of the recruitment stretch and the collagen density is governed by a set of two evolution equations, containing two rate constants.

In our stability analysis we consider the homeostatic equilibrium state as an intermediate state $\mathcal{G}_{I}$ and we superimpose a small dynamic perturbation on this state. The perturbed state is out of equilibrium because the collagen stretch deviates from $\lambda_{c}^{\text {hom }}$. Whether, via dynamic remodeling, the artery will recover its intermediate equilibrium state depends on the rate constants. Thus, the condition of stability of the healthy state against remodeling limits the range of physiologically acceptable values of the rate constants.

In Sect. 2, we recapitulate the model of [15] and list the relations for deformations and stresses in the tissue. In Sect. 3, we consider a pressurized healthy artery and present an analytical solution in the homeostatic equilibrium state $\mathcal{G}_{I}$ for both a thick-walled and a thin-walled artery. The linear stability analysis of the evolution equations 
is presented in Sect. 4 and the result in terms of an explicit stability criterion is derived in Sect. 5. Finally, some conclusions are given in Sect. 6.

\section{Tissue mechanics and remodeling}

\subsection{Deformations and stresses in the tissue}

In this section we present the mechanics part of the model. The membrane formulation of [15] is replaced here by a general 3D description of tissue mechanics.

The tissue is represented by a continuous material body $\mathscr{B}$ occupying at the initial moment of time, at which the body is in its undeformed stress-free state, a three-dimensional region of Euclidean space $\mathbb{R}^{3}$ referred to as the reference configuration $\mathcal{G}_{R}$. During its motion, the body occupies time-dependent actual configurations $\mathcal{G}=\mathcal{G}(t)$. The reference position vector of a material point $\mathscr{P} \in \mathscr{B}$ in configuration $\mathcal{G}_{R}$ is denoted as $\mathbf{X}$. The actual position vector $\mathbf{x} \in \mathcal{G}$ is described by a bijective function $\mathbf{x}=\mathbf{x}(\mathbf{X}, t)$, and the associated deformation is described by the deformation gradient

$\mathcal{F}=\frac{\partial \mathbf{x}}{\partial \mathbf{X}}$.

The tissue is assumed to be incompressible, and composed of an isotropic matrix and collagen fibers, represented by two fiber directions at each material point.

The tissue stretch $\lambda=\lambda(\mathbf{x}, t)$ in the direction $\mathbf{e}_{0}$ is defined by

$\lambda^{2}=\mathcal{F} \mathbf{e}_{0} \cdot \mathcal{F} \mathbf{e}_{0}=\mathbf{e}_{0} \cdot \mathcal{C} \mathbf{e}_{0}$,

where $\mathbf{e}_{0}$ is an arbitrary unit vector in the tissue in the undeformed state $\mathcal{G}_{R}$, and $\mathcal{C}=\mathcal{F}^{T} \mathcal{F}$ is the right CauchyGreen deformation tensor. The introduced tissue stretch $\lambda$ refers to the stretch of the matrix. The deformation of collagen fibers is described by the stretches $\lambda_{c, i}=\lambda_{c, i}(\mathbf{x}, t)$ in the directions $\mathbf{e}_{c, i, 0}, i=1,2$, where $\mathbf{e}_{c, i, 0}$ is a unit vector in the direction of the $i$ th collagen fiber in the reference configuration. Assuming that the collagen fibers deform together with the matrix, we infer that the actual direction of this collagen fiber is given by the unit vector $\mathbf{e}_{c, i}=\mathbf{e}_{c, i}(\mathbf{x}, t)$, defined as

$\mathbf{e}_{c, i}=\frac{1}{\lambda_{f, i}} \mathcal{F} \mathbf{e}_{c, i, 0}$,

where $\lambda_{f, i}=\lambda_{f, i}(\mathbf{x}, t)$ is the tissue stretch in the direction of this fiber. The collagen stretch is different from the tissue stretch because collagen fibers may remain undulated, and therefore unstretched, while tissue is already stretched. The amount of undulation is quantified by the recruitment stretch $\lambda_{\text {rec }}$, which is the stretch of the tissue in the direction of the collagen fiber needed to make the fiber straight but unstretched. Any further increase of the stretch of the tissue in the direction of the fiber would result in stretching of the collagen as well. The recruitment stretch $\lambda_{\text {rec }}$ is defined as the ratio between the tissue and the collagen stretch. During the deformation $\lambda_{\text {rec }}$ can change due to remodeling. Therefore, the actual recruitment stretch is a field variable: $\lambda_{\text {rec }, i}=\lambda_{\text {rec }, i}(\mathbf{x}, t)$. The collagen stretch is thus governed by $(i=1,2)$

$\lambda_{c, i}= \begin{cases}1, & \lambda_{f, i} \leq \lambda_{\text {rec }, i}, \\ \frac{\lambda_{f, i}}{\lambda_{\text {rec }, i},} & \lambda_{f, i}>\lambda_{\text {rec }, i},\end{cases}$

where both the collagen stretch $\lambda_{c, i}=\lambda_{c, i}(\mathbf{x}, t)$ and the tissue stretch $\lambda_{f, i}(\mathbf{x}, t)$ are in the direction of the $i$ th collagen fiber $\mathbf{e}_{c, 0, i}$.

Following [15], the strain-energy-density function (SEDF) $w$ of the tissue is given by

$$
w=\frac{1}{2} c_{m}\left(\lambda_{1}^{2}+\lambda_{2}^{2}+\lambda_{3}^{2}-3\right)+\sum_{i=1}^{2} n_{c, i} \frac{k_{1}}{k_{2}}\left[\mathrm{e}^{k_{2}\left(\lambda_{c, i}^{2}-1\right)^{2}}-1\right],
$$


where $c_{m}$ is the shear modulus of the matrix, $\lambda_{i}$ are the principal tissue stretches, $\lambda_{c, i}$ the collagen stretch along the current collagen fiber direction $\mathbf{e}_{c, i}$, which is related to the reference fiber direction $\mathbf{e}_{c, i, 0}$ via (3), $n_{c, i}=n_{c, i}(\mathbf{x}, t)$ the collagen fiber density relative to the density in the reference state, and $k_{1}$ and $k_{2}$ are material parameters of the collagen fibers. Here we deviate slightly from [15] in that we scale $n_{c, i}$ with respect to its value in $\mathcal{G}_{R}$, whereas Watton et al. [15] scale to the value of $n_{c, i}$ in the healthy state. This has some repercussions on the value of $k_{1}$, but we will take care of it further on. In (5), the first term on the right-hand side corresponds to an isotropic neo-Hookean material, simulating the contribution of the matrix. The second term describes the highly nonlinear mechanical response of the two collagen fibers. The fibers display a non-zero stress response only to tensile stretch in the direction of the fibers, provided that they are not undulated.

The Cauchy stress, derived from (5) is given by

$\mathcal{T}=-p \mathcal{I}+c_{m}(\mathcal{B}-\mathcal{I})+\sum_{i=1}^{N} n_{c, i} \tau_{f, i} \mathbf{e}_{c, i} \otimes \mathbf{e}_{c, i}$,

where $p=p(\mathbf{x}, t)$ is the pressure term, originating from the incompressibility of the tissue, $\mathcal{B}=\mathcal{F F}^{T}$ is the left Cauchy-Green deformation tensor, $\mathcal{I}$ is the second-order unit tensor, and $\tau_{f, i}$ is the stress in the collagen fiber defined by

$\tau_{f, i}=2 k_{1} \lambda_{c, i}^{2}\left(\lambda_{c, i}^{2}-1\right) \mathrm{e}^{k_{2}\left(\lambda_{c, i}^{2}-1\right)^{2}}$.

We assume the problem to be quasi-stationary, and the body forces negligibly small. Under these assumptions, the mechanics of the problem is governed by the equilibrium equation

$\operatorname{div} \mathcal{T}=\mathbf{0}$.

The incompressibility of the tissue dictates

$\operatorname{det} \mathcal{F}=1$,

which can be regarded as a relation for the unknown $p$ in (6).

An equilibrium equation must be provided with the appropriate boundary conditions. In general, they will consist of the kinematic boundary conditions

$\mathbf{u}(\mathbf{x}, t)=\mathbf{u}_{p}(\mathbf{x}, t)$,

on one part of the boundary of the tissue, and the dynamic or natural boundary conditions on the other

$\mathcal{T} \mathbf{n}=\mathbf{t}(\mathbf{x}, t)$,

where $\mathbf{u}=\mathbf{x}-\mathbf{X}$ is the displacement vector; $\mathbf{u}_{p}$ and $\mathbf{t}$ are prescribed displacements and tractions at the boundary, respectively, and $\mathbf{n}$ is the unit outward normal to the boundary.

\subsection{Remodeling of the arterial tissue}

Collagen experiences constant turnover: old fibers decay and are replaced by newly deposited fibers. In the homeostatic state, properties of the collagen and its total mass remain constant. When the artery is not in an equilibrium state, turnover aims not only to renew the collagen, but also to restore the homeostasis. The deposited collagen fibers attach to the tissue with the constant pre-stretch $\lambda_{c}^{\text {hom }}$, which we call the homeostatic collagen stretch (Watton et al. [15] call it attachment stretch). The stretch of existing fibers may differ from $\lambda_{c}^{\text {hom }}$, meaning that their recruitment stretch differs from that of the new fibers as well. Watton et al. [15] capture this effect of deposition and degradation

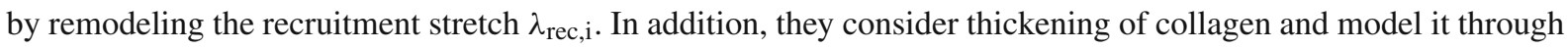
a change in collagen density $n_{c, i}$. The rates of change of the recruitment stretch and collagen density depend on 
the stretch of collagen fibers with respect to the attachment value $\lambda_{c}^{\text {hom }}$, as simulated by the following two linear evolution equations ${ }^{1}$ :

$$
\begin{aligned}
& \frac{\partial \lambda_{\text {rec }, i}}{\partial t}=\alpha\left(\lambda_{c, i}-\lambda_{c}^{\text {hom }}\right), \\
& \frac{\partial n_{c, i}}{\partial t}=\beta\left(\lambda_{c, i}-\lambda_{c}^{\text {hom }}\right), \quad \alpha, \beta \geq 0,
\end{aligned}
$$

where $\lambda_{\text {rec }, i}$ and $n_{c, i}$ are the recruitment stretch and the density of collagen in the $i$ th fiber direction, respectively, and $\alpha$ and $\beta$ are rate constants. The initial conditions for these evolution equations are

$\lambda_{\text {rec }, i}(\mathbf{x}, 0)=\lambda_{\text {rec }, 0, i}, \quad n_{c, i}(\mathbf{x}, 0)=n_{c, 0, i}$.

With this, the recapitulation of the remodeling part of the model of [15] is complete. Next, we derive some trivial relations needed for our further analysis.

By dividing the first equation of (12) by $\alpha$, and the second by $\beta$, and subtracting the resulting equations, we obtain

$\frac{\partial}{\partial t}\left[\frac{1}{\alpha} \lambda_{\text {rec }, i}-\frac{1}{\beta} n_{c, i}\right]=0$.

Integrating this result with respect to time, using the initial conditions (13), we arrive at the first integral of the evolution equations (or conservation equation)

$\frac{\lambda_{\text {rec }, i}}{\alpha}-\frac{n_{c, i}}{\beta}=\frac{\lambda_{\text {rec }, 0, i}}{\alpha}-\frac{n_{c, 0, i}}{\beta}$,

which enables us to directly express $n_{c, i}$ in terms of $\lambda_{\text {rec }, i}$, according to

$n_{c, i}=\frac{\beta}{\alpha}\left(\lambda_{\text {rec }, i}-\lambda_{\text {rec }, 0, i}\right)+n_{c, 0, i}$.

In the following section, we will use the equations introduced in this and the preceding section to describe the inflation of a healthy blood vessel under internal pressure, taking account of the remodeling of the tissue.

\section{Mechanics of the pressurized healthy artery}

In this section, we formulate and solve the problem of static equilibrium of a pressurized healthy artery.

The reference configuration $\mathcal{G}_{R}$ of the artery is a cylindrical tube of inner and outer radii $R_{i}$ and $R_{O}$, respectively. The tissue is described by the constitutive equation (6) and the incompressibility condition (9), while mechanical equilibrium is governed by (8).

Watton et al. [15] model the media and the adventitia (the two mechanically relevant layers of an artery) as separate layers with different material coefficients for each layer. However, for their mechanics model they use a membrane model, in which the SEDF is the sum of the SEDFs of the two layers, leading to an elastic model the stiffness of which is a weighted average of that of the two layers. In our study we simplify this structure by considering one layer only with material parameters equal to weighted-average parameters of the media and the adventitia. In this respect, our one-layer model corresponds with the membrane model of [15]. This layer consists of the matrix with embedded two sets of collagen fibers, arranged symmetrically with respect to the circumferential direction with an angle $2 \gamma$ between them; see Fig. 1. Therefore, the coefficients of the SEDF (5) are related to the coefficients of the SEDFs introduced in [15, Eqs. 30, 31] as follows

$k_{2}=\frac{a_{x}}{4}, \quad k_{1}=\frac{a_{x}}{4}\left(\frac{2}{3} k_{M}+\frac{1}{3} k_{A}\right), \quad c_{m}=k_{g}+\frac{2}{3} k_{E}$,

\footnotetext{
1 Watton et al. [15] introduce in their Eq. 32 the evolution equations in a slightly different form, in so far that they use the strains $(\varepsilon)$ instead of the stretches $(\lambda)$. However, in a linear formulation (assuming $\varepsilon \ll 1$ and $\lambda=1+O(\varepsilon) \approx 1$ ) these two formulations become identical up to $O\left(\varepsilon^{2}\right)$.
} 
Fig. 1 Schematic representation of the artery, consisting of the matrix with embedded collagen fibers, arranged in two helices with the angle $2 \gamma$ between them

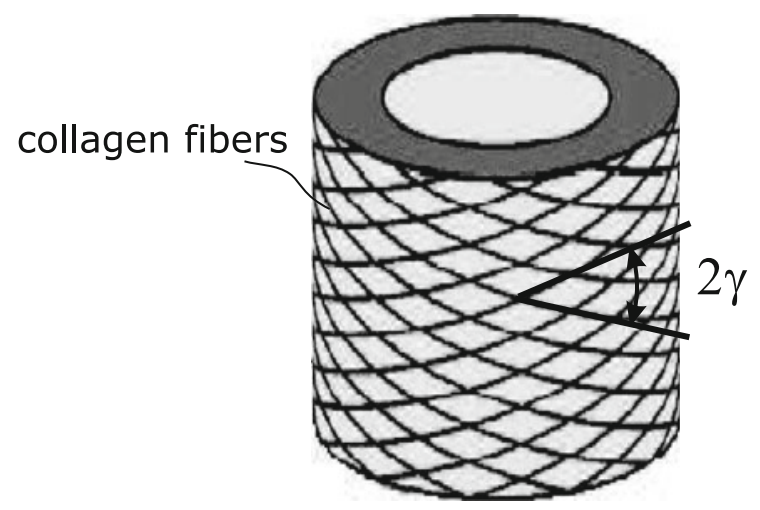

with $a_{x}, k_{M}, k_{A}, k_{g}$ and $k_{E}$ the material parameters in [15]. We note that the stiffness of a collagen fiber is governed by $n_{c} k_{1}$ (see (6) and (7)). In [15], the relative collagen density $n_{c}$ is defined with respect to the healthy state, thus $n_{c, H}=1$, where the subscript $H$ denotes the healthy state. In our approach we do not know the healthy state beforehand, and we define $n_{c}$ with respect to the initial state, setting $n_{c, 0}=1$. In order to keep the stiffness at the healthy state the same as in [15] we divide $k_{1}$, obtained from (17), by an estimate for $n_{c, H}$.

Neglecting the influence of the surrounding tissue, we assume that the outer surface of the artery is traction-free. The inner surface is loaded by a prescribed blood pressure $p_{\mathrm{I}}$. This leads us to the boundary conditions

$\left.\sigma_{r r}\right|_{R=R_{i}}=-p_{\mathrm{I}},\left.\quad \sigma_{r r}\right|_{R=R_{o}}=0$,

with $\sigma_{r r}$ the radial normal-stress component of the stress tensor $\mathcal{T}$. In addition to that, the artery is stretched in the axial direction by a prescribed uniform stretch $\lambda_{z}$.

Since the artery is uniformly stretched in the axial direction and uniformly loaded by the internal pressure, the collagen fiber directions remain symmetric, and as the matrix is isotropic, the resulting problem is rotationally symmetric. Moreover, all the parameters and variables associated with the two fiber directions are the same for both fibers, i.e.,

$$
\begin{aligned}
& n_{c, 0,1}=n_{c, 0,2} \equiv n_{c, 0}, \quad \lambda_{\text {rec }, 0,1}=\lambda_{\text {rec }, 0,2} \equiv \lambda_{\text {rec }, 0} \\
& n_{c, 1}=n_{c, 2} \equiv n_{c}, \quad \lambda_{\text {rec }, 1}=\lambda_{\text {rec }, 2} \equiv \lambda_{\text {rec }} \\
& \lambda_{f, 1}=\lambda_{f, 2} \equiv \lambda_{f}, \quad \tau_{f, 1}=\tau_{f, 2}=\tau_{f} .
\end{aligned}
$$

Consequently, Eq. 12 is reduced to two equations on $n_{c}$ and $\lambda_{\text {rec }}$, and each of the relations (14)-(16) is reduced to one equation.

\subsection{Thick-walled artery}

The position of a material point in the stress-free undeformed reference state $\mathcal{G}_{R}$ is given by the cylindrical coordinates $(R, \Theta, Z)$, while the position of the same point in the intermediate pressurized and deformed state $\mathcal{G}_{I}$ is given by $(r, \theta, z)$. Due to the symmetry of the problem we have $r=r(R), \theta=\Theta$, and $z=\lambda_{z} Z$. Since there are no shears involved, we have $\left\{\mathbf{e}_{r}, \mathbf{e}_{\theta}, \mathbf{e}_{z}\right\}=\left\{\mathbf{e}_{R}, \mathbf{e}_{\Theta}, \mathbf{e}_{Z}\right\}$. Taking this into account, we obtain for the deformation gradient

$\mathcal{F}=\lambda_{r} \mathbf{e}_{R} \otimes \mathbf{e}_{R}+\lambda_{\theta} \mathbf{e}_{\Theta} \otimes \mathbf{e}_{\Theta}+\lambda_{z} \mathbf{e}_{Z} \otimes \mathbf{e}_{Z}=\frac{\mathrm{d} r}{\mathrm{~d} R} \mathbf{e}_{R} \otimes \mathbf{e}_{R}+\frac{r}{R} \mathbf{e}_{\Theta} \otimes \mathbf{e}_{\Theta}+\lambda_{z} \mathbf{e}_{Z} \otimes \mathbf{e}_{Z}$,

with $\lambda_{r}$ and $\lambda_{\theta}$ the radial and the circumferential components of the deformation gradient, respectively.

The unit vectors $\mathbf{e}_{c, 1,0}$ and $\mathbf{e}_{c, 2,0}$, coinciding with the original (reference) directions of collagen fibers are given by

$\mathbf{e}_{c, 1,0}=\cos \gamma \mathbf{e}_{\Theta}+\sin \gamma \mathbf{e}_{Z}, \quad \mathbf{e}_{c, 2,0}=\cos \gamma \mathbf{e}_{\Theta}-\sin \gamma \mathbf{e}_{Z}$. 
Taking the Euclidian norm of both sides of (3) and using (19), we find the tissue stretch in the direction of the fibers as

$\lambda_{f}=\left\|\mathcal{F} \mathbf{e}_{c, 1,0}\right\| \equiv\left\|\mathcal{F} \mathbf{e}_{c, 2,0}\right\|, \quad i=1,2$,

or with (21) and (20),

$\lambda_{f}=\sqrt{\lambda_{\theta}^{2} \cos ^{2} \gamma+\lambda_{z}^{2} \sin ^{2} \gamma}$.

Substituting (21) and (20) in (3), we find the unit vectors of the collagen fibers in the actual configuration as

$\mathbf{e}_{c, 1}=\frac{\lambda_{\theta}}{\lambda_{f}} \cos \gamma \mathbf{e}_{\Theta}+\frac{\lambda_{z}}{\lambda_{f}} \sin \gamma \mathbf{e}_{Z}, \quad \mathbf{e}_{c, 2}=\frac{\lambda_{\theta}}{\lambda_{f}} \cos \gamma \mathbf{e}_{\Theta}-\frac{\lambda_{z}}{\lambda_{f}} \sin \gamma \mathbf{e}_{Z}$.

The incompressibility condition (9) yields

$\lambda_{z} \frac{r}{R} \frac{\mathrm{d} r}{\mathrm{~d} R}=1$,

having the solution

$r(R)=\sqrt{\frac{R^{2}+k R_{i}^{2}}{\lambda_{z}}}, \quad k>0$,

which determines the current radial coordinate $r$ of a material point of the cylinder wall as a function of the reference coordinate $R$ and an unknown dimensionless constant $k$. This constant will be determined from the equilibrium equation (8) and the boundary conditions (18). For this, we need the equilibrium equation (8) in cylindrical coordinates. Taking into account that all shear stresses are zero and the only relevant components of $\mathcal{T}$ are the normal stresses $\sigma_{r r}$ and $\sigma_{\theta \theta}$, which are functions of $r$ only (also $\sigma_{z z} \neq 0$, but not relevant here), the equilibrium equation in cylindrical coordinates reads

$\frac{\mathrm{d} \sigma_{r r}}{\mathrm{~d} r}+\frac{1}{r}\left(\sigma_{r r}-\sigma_{\theta \theta}\right)=0$.

With the boundary conditions (18) we can integrate (27) over the wall thickness of the artery to obtain $\left(r_{i, o}=\right.$ $\left.r\left(R_{i, o}\right)\right)$

$\int_{r_{i}}^{r_{o}} \frac{\mathrm{d} \sigma_{r r}}{\mathrm{~d} r} \mathrm{~d} r=p_{\mathrm{I}}=-\int_{r_{i}}^{r_{o}}\left(\sigma_{r r}(r)-\sigma_{\theta \theta}(r)\right) \frac{\mathrm{d} r}{r}=\int_{R_{i}}^{R_{o}}\left(\tilde{\sigma}_{\theta \theta}(R)-\tilde{\sigma}_{r r}(R)\right) \frac{R}{R^{2}+k R_{i}^{2}} \mathrm{~d} R$,

where $\tilde{\sigma}(R)=\sigma(r(R))$, according to (26).

The left Cauchy-Green strain tensor follows from (20) and (26) as

$$
\begin{aligned}
\mathcal{B} & =\mathcal{F} \mathcal{F}^{T}=\lambda_{r}^{2} \mathbf{e}_{R} \otimes \mathbf{e}_{R}+\lambda_{\theta}^{2} \mathbf{e}_{\Theta} \otimes \mathbf{e}_{\Theta}+\lambda_{z}^{2} \mathbf{e}_{Z} \otimes \mathbf{e}_{Z} \\
& =\left(\frac{\hat{R}^{2}}{\lambda_{z}\left(\hat{R}^{2}+k\right)}\right) \mathbf{e}_{R} \otimes \mathbf{e}_{R}+\left(\frac{\hat{R}^{2}+k}{\lambda_{z} \hat{R}^{2}}\right) \mathbf{e}_{\Theta} \otimes \mathbf{e}_{\Theta}+\lambda_{z}^{2} \mathbf{e}_{Z} \otimes \mathbf{e}_{Z},
\end{aligned}
$$

where $\hat{R}=R / R_{i}$ (from here on we scale all length parameters on $R_{i}$, and we omit the hat together with the tilde on $\sigma$ ). From the constitutive relation (6) we then obtain

$$
\begin{aligned}
\sigma_{r r} & =-p+c_{m}\left(\frac{R^{2}}{\lambda_{z}\left(R^{2}+k\right)}-1\right), \\
\sigma_{\theta \theta} & =-p+c_{m}\left(\frac{R^{2}+k}{\lambda_{z} R^{2}}-1\right)+\sum_{i=1,2} n_{c, i}\left(\mathbf{e}_{\Theta} \cdot \mathcal{T}_{c, i} \mathbf{e}_{\Theta}\right),
\end{aligned}
$$

where $\mathcal{T}_{c, i}=\tau_{f, i} \mathbf{e}_{c, i} \otimes \mathbf{e}_{c, i}$. With (24) we derive

$\mathbf{e}_{\Theta} \cdot \mathcal{T}_{c, 1} \mathbf{e}_{\Theta}=\mathbf{e}_{\Theta} \cdot \mathcal{T}_{c, 2} \mathbf{e}_{\Theta}=\tau_{f}^{\text {hom }}\left(\frac{\lambda_{\theta}}{\lambda_{f}}\right)^{2} \cos ^{2} \gamma=\tau_{f}^{\text {hom }} \frac{\lambda^{2}(R)}{\lambda^{2}(R)+\lambda_{z}^{2} \tan ^{2} \gamma}$, 
where

$\lambda(R)=\lambda_{\theta}(R)=\frac{1}{R} \sqrt{\frac{R^{2}+k}{\lambda_{z}}}$,

and

$\tau_{f, 1}=\tau_{f, 2}=\tau_{f}^{\text {hom }} \equiv \tau_{f}\left(\lambda_{c}^{\text {hom }}\right)=2 k_{1}\left[\lambda_{c}^{\text {hom }}\right]^{2}\left(\left[\lambda_{c}^{\text {hom }}\right]^{2}-1\right) \mathrm{e}^{k_{2}\left(\left[\lambda_{c}^{\text {hom }}\right]^{2}-1\right)^{2}}$,

according to (7) with $\lambda_{c, 1}=\lambda_{c, 2}=\lambda_{c}^{\text {hom }}$.

Substitution of (19) in an equilibrium state in (16) gives

$$
\begin{aligned}
n_{c}(R) & =\frac{\beta}{\alpha}\left(\lambda_{\text {rec }}(R)-\lambda_{\text {rec }, 0}\right)+n_{c, 0}=\frac{\beta}{\alpha}\left(\frac{\lambda_{f}(R)}{\lambda_{c}}-\lambda_{\text {rec }, 0}\right)+n_{c, 0} \\
& =\frac{\beta}{\alpha}\left(\frac{\cos \gamma}{\lambda_{c}^{\text {hom }}} \sqrt{\lambda^{2}(R)+\lambda_{z}^{2} \tan ^{2} \gamma}-\lambda_{\text {rec }, 0}\right)+n_{c, 0},
\end{aligned}
$$

where we have successively used (4) 2 and (23).

With these results, we can write out $(30)_{2}$ as

$$
\begin{aligned}
\sigma_{\theta \theta}(R)= & -p(R)+c_{m}\left(\frac{R^{2}+k}{\lambda_{z} R^{2}}-1\right) \\
& +2 \tau_{f}^{\text {hom }}\left[\frac{\beta}{\alpha}\left(\frac{\cos \gamma}{\lambda_{c}^{\text {hom }}} \sqrt{\lambda^{2}(R)+\lambda_{z}^{2} \tan ^{2} \gamma}-\lambda_{\text {rec }, 0}\right)+n_{c, 0}\right] \frac{\lambda^{2}(R)}{\lambda^{2}(R)+\lambda_{z}^{2} \tan ^{2} \gamma} .
\end{aligned}
$$

Substituting (30) and (35) in (28) and evaluating the resulting integral, we arrive at

$$
\begin{aligned}
p_{\mathrm{I}}= & \frac{c_{m}}{2 \lambda_{z}}\left(\frac{k\left(R_{o}^{2}-1\right)}{\left(R_{o}^{2}+k\right)(1+k)}+\log \frac{R_{o}^{2}(1+k)}{\left(R_{o}^{2}+k\right)}\right) \\
& +\frac{\tau_{f}^{\text {hom }}}{w}\left[\frac{2 \beta \cos \gamma}{\alpha \lambda_{c}^{\text {hom }}} \sqrt{\frac{w}{\lambda_{z}}} \log \frac{R_{o} \sqrt{w}+\sqrt{R_{o}^{2} w+k}}{\sqrt{w}+\sqrt{w+k}}-\left(\frac{\beta}{\alpha} \lambda_{\text {rec }, 0}-n_{c, 0}\right) \log \frac{w R_{o}^{2}+k}{w+k}\right],
\end{aligned}
$$

where $w=\sqrt{1+\lambda_{z}^{3} \tan ^{2} \gamma}$.

Once $k$ is found from the latter relation, the current coordinate $r$ can be determined from (26), and the tissue stretches from (20).

\subsection{Thin-walled artery}

Here, we will reduce (36) for the limiting case of a thin-walled artery. The outer radius can be written as

$$
\frac{R_{o}}{R_{i}}=\hat{R}_{o}=\left(1+\frac{H}{R_{i}}\right)=1+\varepsilon,
$$

with $H$ the thickness of the cylinder, being small as compared to the inner radius: $\varepsilon=H / R_{i} \ll 1$. From now on, we will neglect terms that are of higher order in $\varepsilon$, and then (36) reduces to

$p_{\mathrm{I}}=\frac{H}{R}\left[\frac{c_{m}}{\lambda_{z}} \frac{k(2+k)}{(1+k)^{2}}+\frac{2 \tau_{f}^{\text {hom }}}{w+k}\left(\frac{\beta \cos \gamma}{\alpha \lambda_{c}^{\text {hom } \sqrt{\lambda_{z}}}} \sqrt{w+k}-\frac{\beta}{\alpha} \lambda_{\text {rec }, 0}+n_{c, 0}\right)\right]$.

Since, according to (29), (26), and (4) 2 , the circumferential stretch and the recruitment stretch are given by

$\lambda=\sqrt{\frac{1+k}{\lambda_{z}}}$, and $\lambda_{\text {rec }}=\frac{\cos \gamma}{\lambda_{c}^{\text {hom }}} \sqrt{\lambda^{2}+\lambda_{z}^{2} \tan ^{2} \gamma}=\frac{\cos \gamma}{\lambda_{c}^{\text {hom } \sqrt{\lambda_{z}}}} \sqrt{w+k}$, 
Fig. 2 Relationship between the blood pressure $p$, and the tissue stretch $\lambda$ during remodeling from an initial state $\mathcal{G}_{0}$ to an intermediate state $\mathcal{G}_{I}$

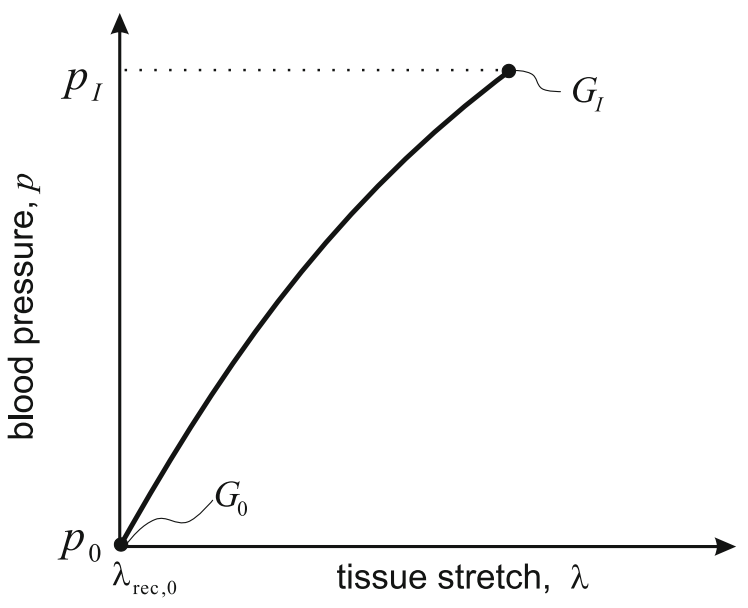

respectively, we can rewrite (38) in terms of the circumferential stretch $\lambda$ and the recruitment stretch $\lambda_{\text {rec }}$ as

$p_{\mathrm{I}}=\frac{H}{\lambda_{z} R}\left[\frac{c_{m}}{\lambda_{z}^{2}}\left(\lambda_{z}^{2}-\frac{1}{\lambda^{4}}\right)+\frac{2 n_{c} \tau_{f}^{\text {hom }}}{\lambda^{2}+\lambda_{z}^{2} \tan ^{2} \gamma}\right]$,

where

$n_{c}=\frac{\beta}{\alpha}\left(\lambda_{\text {rec }}-\lambda_{\text {rec }, 0}\right)+n_{c, 0}$.

We will use (40) for our stability analysis in the next section.

\section{Stability of the healthy artery}

In this section, we investigate the stability against remodeling of the intermediate state $\mathcal{G}_{I}$ of the artery described in the preceding section. The intermediate state of the tissue is obtained from the initial state $\mathcal{G}_{0}$ via remodeling as described in the next subsection.

\subsection{Remodeling in the approach to the intermediate state}

If $\lambda_{c}=\lambda_{c}^{\text {hom }}$ throughout the artery, then there is no remodeling, and we say that the artery is in homeostatic equilibrium. The initial state $\mathcal{G}_{0}$ of the artery is supposed to be a homeostatic state. We choose the initial recruitment $\lambda_{\text {rec, } 0}$ and initial volume fraction of collagen $n_{c, 0}$ at the state $\mathcal{G}_{0}$ such that equilibrium is realized under a pressure $p_{0}$, which is much lower than the physiological pressure $p_{\mathrm{ph}}$. If the pressure increases to some intermediate value $p_{\mathrm{I}}$, the collagen stretches beyond $\lambda_{c}^{\text {hom }}$. This causes violation of the equilibrium, and, consequently, collagen remodeling. Assuming that the pressure increases very slowly with respect to the remodeling speed, the tissue reaches the intermediate state $\mathcal{G}_{I}$, see Fig. 2. We will now investigate what the conditions are under which the tissue will reach a stable intermediate state $\mathcal{G}_{I}$.

\subsection{Stability analysis of the evolution equations for a thin-walled artery}

We first consider the thin-walled artery, in which mechanical equilibrium of the tissue at the current time $t$ in the perturbed state is described by the following equations (which follow successively from (4), (7), (16), (19), (23), and (40)) 
$\Pi\left(\lambda, \lambda_{\text {rec }}\right) \equiv \frac{1}{\lambda_{z}^{2}}\left(\lambda_{z}^{2}-\frac{1}{\lambda^{4}(t)}\right)+\frac{2 n_{c}(t)}{\lambda^{2}(t)+\lambda_{z}^{2} \tan ^{2} \gamma} \hat{\tau}_{f}\left(\lambda_{c}(t)\right)=\hat{p}_{\mathrm{I}} \equiv \frac{\lambda_{z} R}{H} \frac{p_{\mathrm{I}}}{c_{m}}$,

where

$\lambda_{c}(t)=\frac{\lambda_{f}(t)}{\lambda_{\text {rec }}(t)}=\frac{\sqrt{\lambda^{2}(t) \cos ^{2} \gamma+\lambda_{z}^{2} \sin ^{2} \gamma}}{\lambda_{\text {rec }}(t)}$,

$n_{c}(t)=\frac{\beta}{\alpha}\left(\lambda_{\text {rec }}(t)-\lambda_{\text {rec }, 0}\right)+n_{c, 0}$,

$\hat{\tau}_{f}\left(\lambda_{c}\right)=\tau_{f}\left(\lambda_{c}\right) / c_{m}=2 \hat{k}_{1} \lambda_{c}^{2}\left(\lambda_{c}^{2}-1\right) \mathrm{e}^{k_{2}\left(\lambda_{c}^{2}-1\right)^{2}}$,

with $\hat{k}_{1}=k_{1} / c_{m}$, and $\lambda_{c}=\lambda_{c, 1}=\lambda_{c, 2}$. The unknowns here are $\lambda(t), \lambda_{c}(t), \lambda_{\text {rec }}(t)$ and $n_{c}(t)$. In addition to (43), $\lambda_{\text {rec }}$ is related to $\lambda_{c}$ through the evolution equation (12), i.e.,

$\frac{\mathrm{d} \lambda_{\text {rec }}}{\mathrm{d} t}(t)=\alpha\left(\lambda_{c}(t)-\lambda_{c}^{\text {hom }}\right)$.

With (43) $)_{1}$ and (43) $)_{2}$, the collagen stretch $\lambda_{c}$ and the collagen density $n_{c}$ can be eliminated from the system. Then only two unknowns are left: the tissue stretch $\lambda$ and the recruitment stretch $\lambda_{\text {rec }}$. The stationary solution of the system (42)-(44), corresponding to the intermediate equilibrium state $\mathcal{G}_{I}$, is given by

$\lambda_{c}=\lambda_{c, I}=\lambda_{c}^{\text {hom }}, \quad \lambda=\lambda_{I}, \quad \lambda_{\text {rec }}=\lambda_{\text {rec }, I}, \quad n_{c}=n_{c, I}=\frac{\beta}{\alpha}\left(\lambda_{\text {rec }, I}-\lambda_{\text {rec }, 0}\right)+n_{c, 0}$,

where $\lambda_{\text {rec }, I}$ follows from (43) 1 as

$\lambda_{\text {rec }, I}=\frac{\lambda_{f, I}}{\lambda_{c}^{\text {hom }}}=\frac{\cos \gamma}{\lambda_{c}^{\text {hom }}} \sqrt{\lambda_{I}^{2}+\lambda_{z}^{2} \tan ^{2} \gamma}$,

while $\lambda_{I}$ is the solution of (42) for given $\hat{p}_{I}$.

The perturbed state can be presented in the form:

$\lambda(t)=\lambda_{I}+\delta \lambda(t), \quad \lambda_{\text {rec }}(t)=\lambda_{\text {rec }, I}+\delta \lambda_{\text {rec }}(t), \quad n_{c}(t)=n_{c, I}+\delta n_{c}(t), \quad \lambda_{c}(t)=\lambda_{c}^{\text {hom }}+\delta \lambda_{c}(t)$,

with $\delta \lambda(t), \delta \lambda_{\text {rec }}(t), \delta n_{c}(t)$, and $\delta \lambda_{c}(t)$ the perturbations (or variations) of the circumferential tissue stretch, the recruitment stretch, the collagen density, and the collagen stretch, respectively. Variations of the tissue stretch and the recruitment stretch are considered to be independent of each other, while the variations of collagen stretch $\lambda_{c}$, collagen density $n_{c}$ and collagen stress $\hat{\tau}_{f}$ can be found successively from (43) $)_{2},(43)_{1}$ and (43) $)_{3}$ as

$$
\begin{aligned}
& \delta n_{c}=\frac{\beta}{\alpha} \delta \lambda_{\text {rec }}, \quad \delta \lambda_{f}=\cos ^{2} \gamma \frac{\lambda}{\lambda_{f}} \delta \lambda, \\
& \delta \lambda_{c}=\frac{\lambda_{f}}{\lambda_{\text {rec }}}\left(\frac{\delta \lambda_{f}}{\lambda_{f}}-\frac{\delta \lambda_{\text {rec }}}{\lambda_{\text {rec }}}\right)=\lambda_{c}^{\text {hom }}\left(\frac{\lambda}{\lambda^{2}+\lambda_{z}^{2} \tan ^{2} \gamma} \delta \lambda-\frac{\delta \lambda_{\text {rec }}}{\lambda_{\text {rec }}}\right), \\
& \delta \hat{\tau}_{f}=2 \hat{\tau}_{f}^{\text {hom }} \tilde{\tau}_{f}^{\text {hom }}\left(\frac{\lambda}{\lambda^{2}+\lambda_{z}^{2} \tan ^{2} \gamma} \delta \lambda-\frac{\delta \lambda_{\text {rec }}}{\lambda_{\text {rec }}}\right),
\end{aligned}
$$

where $\hat{\tau}_{f}^{\text {hom }}=\hat{\tau}_{f}\left(\lambda_{c}^{\text {hom }}\right)$ and

$$
\tilde{\tau}_{f}^{\text {hom }}=\frac{2\left[\lambda_{c}^{\text {hom }}\right]^{2}-1}{\left[\lambda_{c}^{\text {hom }}\right]^{2}-1}+2 k_{2}\left[\lambda_{c}^{\text {hom }}\right]^{2}\left(\left[\lambda_{c}^{\text {hom }}\right]^{2}-1\right) .
$$

These relations must be evaluated for $\lambda=\lambda_{I}$, and $\lambda_{\text {rec }}=\lambda_{\text {rec }, I}$. Using these partial results, we find from the first variation of $\Pi$ (notice that $p_{\mathrm{I}}$ is fixed)

$$
\delta \Pi=\delta \Pi\left(\lambda, \lambda_{\text {rec }}\right)=0, \Rightarrow\left(\frac{\partial \Pi}{\partial \lambda}\right)_{I} \delta \lambda+\left(\frac{\partial \Pi}{\partial \lambda_{\text {rec }}}\right)_{I} \delta \lambda_{\text {rec }}=0,
$$

that 
$\delta \lambda=L \delta \lambda_{\text {rec }}$,

with

$L=\frac{A}{B}$,

and

$$
\begin{aligned}
& A=-\left(\frac{\partial \Pi}{\partial \lambda_{\text {rec }}}\right)_{I}=-\frac{2 \hat{\tau}_{f}^{\text {hom }}}{\lambda^{2}+\lambda_{z}^{2} \tan ^{2} \gamma}\left[\frac{\beta}{\alpha}-\frac{2 n_{c} \tilde{\tau}_{f}^{\text {hom }}}{\lambda_{\text {rec }}}\right], \\
& B=\left(\frac{\partial \Pi}{\partial \lambda}\right)_{I}=\frac{4}{\lambda_{z}^{2} \lambda^{5}}+\frac{4 n_{c} \hat{\tau}_{f}^{\text {hom }} \lambda}{\left(\lambda^{2}+\lambda_{z}^{2} \tan ^{2} \gamma\right)^{2}}\left(\tilde{\tau}_{f}^{\text {hom }}-1\right) .
\end{aligned}
$$

In the expressions above, one must take $\lambda=\lambda_{I}, \lambda_{\text {rec }}=\lambda_{\text {rec }, I}$, and $n_{c}=n_{c, I}$, according to (45) and (46). Taking the first variation of (44), and substituting $(48)_{2},(43)_{1}$ and (51) in the result, we get the equation for the variation $\delta \lambda_{\text {rec }}$

$$
\frac{\mathrm{d}}{\mathrm{d} t} \delta \lambda_{\mathrm{rec}}(t)=\frac{\alpha \lambda_{c}^{\text {hom }}}{\lambda^{2}+\lambda_{z}^{2} \tan ^{2} \gamma}\left(\lambda L-\frac{\lambda_{c}^{\text {hom }}}{\cos \gamma} \sqrt{\lambda^{2}+\lambda_{z}^{2} \tan ^{2} \gamma}\right) \delta \lambda_{\text {rec }}(t),
$$

with the solution

$$
\delta \lambda_{\text {rec }}(t)=C_{1} \exp \left\{\frac{\alpha \lambda_{c}^{\text {hom }}}{\lambda^{2}+\lambda_{z}^{2} \tan ^{2} \gamma}\left(\lambda L-\frac{\lambda_{c}^{\text {hom }}}{\cos \gamma} \sqrt{\lambda^{2}+\lambda_{z}^{2} \tan ^{2} \gamma}\right) t\right\},
$$

where $C_{1}$ is an arbitrary but irrelevant constant.

The intermediate state $\mathcal{G}_{I}$ is stable if and only if the perturbations decay with time, so $\lim _{t \rightarrow \infty} \delta \lambda_{\text {rec }}(t)=0$. In this case, also $\delta \lambda(t)$ tends to zero. Hence, according to (56), we formulate the stability against remodeling of the intermediate equilibrium state $\mathcal{G}_{I}$ under given pressure $p_{\mathrm{I}}$ by the following

\subsubsection{Statement}

If

$L=L\left(\lambda ; \frac{\beta}{\alpha}\right)=\left\{\begin{array}{l}<\frac{\lambda_{c}^{\text {hom }}}{\lambda \cos \gamma} \sqrt{\lambda^{2}+\lambda_{z}^{2} \tan ^{2} \gamma}=: L_{\mathrm{cr}}, \quad \text { the solution is stable, } \\ >\frac{\lambda_{c}^{\text {hom }}}{\lambda \cos \gamma} \sqrt{\lambda^{2}+\lambda_{z}^{2} \tan ^{2} \gamma}, \quad \text { the solution is unstable, }\end{array}\right.$

where $\lambda$ follows from (see (42), (43) 2 )

$\Pi_{I}\left(\lambda ; \frac{\beta}{\alpha}\right) \equiv 1-\frac{1}{\lambda_{z}^{2} \lambda^{4}}+\left[\frac{\beta}{\alpha}\left(\frac{\Lambda(\lambda)}{\lambda_{c}^{\text {hom }}} \cos \gamma-\lambda_{\text {rec }, 0}\right)+n_{c, 0}\right] \frac{2 \hat{\tau}_{f}^{\text {hom }}}{\Lambda^{2}(\lambda)}=\hat{p}_{\mathrm{I}}$,

where $\Lambda(\lambda)=\sqrt{\lambda^{2}+\lambda_{z}^{2} \tan ^{2} \gamma}$.

From this statement we can obtain an allowable range of $(\beta / \alpha)$-values for which the homeostatic equilibrium state $\mathcal{G}_{H}\left(p=p_{\mathrm{ph}}\right)$ is stable by the following formal procedure:

Find the critical $(\beta / \alpha)$-value from (57), (58) for given $\hat{p}_{\mathrm{I}}$ by numerically solving $L(\lambda ; \beta / \alpha)=L_{\mathrm{cr}}$ and $\Pi_{I}(\lambda ; \beta / \alpha)=\hat{p}_{\mathrm{I}}$. The computed value $(\beta / \alpha)_{\mathrm{cr}}$ is a function of $\hat{p}_{\mathrm{I}}$, and the function $(\beta / \alpha)_{\mathrm{cr}}\left(\hat{p}_{\mathrm{I}}\right)$ is monotone increasing with $\hat{p}_{\mathrm{I}}$ (see Fig. 3 further on). Since $\hat{p}_{\mathrm{I}} \leqslant \hat{p}_{\mathrm{ph}}$, the maximum value for $(\beta / \alpha)_{\text {cr }}$ occurs for $\hat{p}_{\mathrm{I}}=\hat{p}_{\mathrm{ph}}$. Hence, for all values of $(\beta / \alpha)>(\beta / \alpha)_{\mathrm{cr}}\left(\hat{p}_{\mathrm{ph}}\right)$, every intermediate equilibrium state $\mathcal{G}_{I}$, with $\hat{p}_{\mathrm{I}} \leq \hat{p}_{\mathrm{ph}}$, is stable against remodeling. 


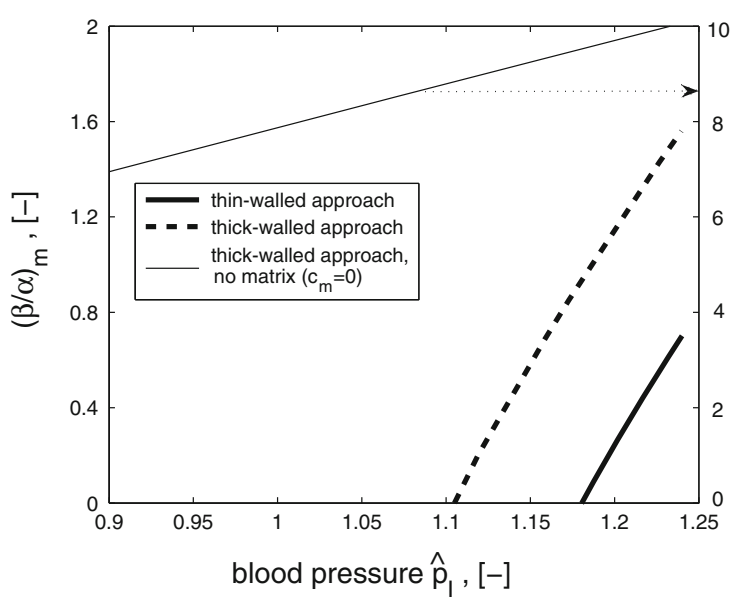

Fig. 3 Dependence of $(\beta / \alpha)_{\mathrm{m}}$ on the intermediate pressure $\hat{p}_{\mathrm{I}}$ for the cerebral artery. Results are depicted for the thin-walled approach (-), the thick-walled approach (- - ), and in case $c_{m}=0(-)$, see Sect. 5.5. Note that for the latter the values of $(\beta / \alpha)_{\mathrm{m}}$ are much higher (for this the right vertical axis is used). Each line on the graph separates the upper region of stability from the lower region of instability

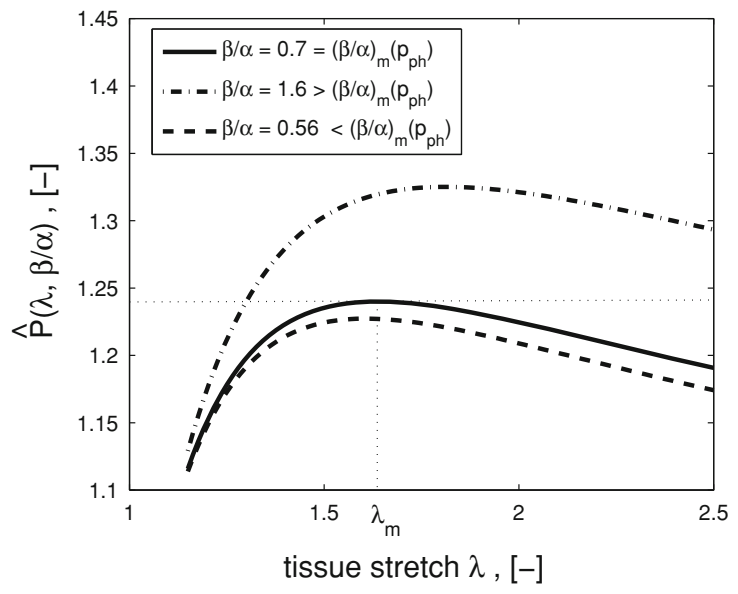

Fig. 4 The function $\hat{P}=\hat{P}(\lambda ; \beta / \alpha)$ according to (59), thinwalled approach, for different values of $\beta / \alpha$. The lines represent the scaled pressure borne by an artery in a homeostatic state having a circumferential stretch $\lambda$

However, we can find and interpret this result also in an alternative way, which yields an easier numerical procedure. We consider the left-hand side of (58) as a function of $\lambda$ and $\beta / \alpha$ according to (we wish to consider this function here as a scaled pressure, and therefore we use the symbol $\hat{P}$ instead of $\Pi_{I}$ )

$\hat{P}\left(\lambda ; \frac{\beta}{\alpha}\right)=1-\frac{1}{\lambda_{z}^{2} \lambda^{4}}+\left[\frac{\beta}{\alpha}\left(\frac{\Lambda(\lambda)}{\lambda_{c}^{\text {hom }}} \cos \gamma-\lambda_{\text {rec }, 0}\right)+n_{c, 0}\right] \frac{2 \hat{\tau}_{f}^{\text {hom }}}{\Lambda^{2}(\lambda)}$.

If we plot $\hat{P}$ as a function of $\lambda$ for different values of $\beta / \alpha$ (see Fig. 4 further on), then we observe that these graphs have a local maximum of pressure $\hat{P}_{\max }$. The tissue stretch at which $\hat{P}_{\max }$ is reached can be found from the relation $\frac{\mathrm{d} \hat{P}_{\mathrm{I}}}{\mathrm{d} \lambda}=0$.

However, if we compare this with (50), we see that this is exactly the same relation (although, maybe, in a quite different interpretation). Let the maximum of $\hat{P}$ be reached for $\lambda=\lambda_{\mathrm{m}}=\lambda_{\mathrm{m}}(\beta / \alpha)$, and let $\hat{P}_{\max }(\beta / \alpha)$ be the maximum, scaled pressure that can be reached, thus

$\hat{P}_{\max }=\hat{P}\left(\lambda_{\mathrm{m}}(\beta / \alpha), \beta / \alpha\right)$.

This means that for a chosen value of $\beta / \alpha$, the pressure can, for any value of $\lambda$, reach only values $\hat{p}<\hat{P}_{\max }$. It turns out that this $\hat{P}_{\text {max }}$ is a monotone increasing function of $\beta / \alpha$ (at least for the values we considered). We can interpret this also the other way around: for each value of $\hat{p}_{\mathrm{I}} \in\left[\hat{p}_{0}, \hat{p}_{\mathrm{ph}}\right]$ there exists $(\beta / \alpha)_{\mathrm{m}}\left(\hat{p}_{\mathrm{I}}\right)$ such that for $\beta / \alpha<(\beta / \alpha)_{\mathrm{m}}$ the pressure cannot reach the value $\hat{p}_{\mathrm{I}}$, whereas for $\beta / \alpha>(\beta / \alpha)_{\mathrm{m}}$ it can reach pressure values up to and higher than $\hat{p}_{\mathrm{I}}$. We obtain the maximum value of $(\beta / \alpha)_{\mathrm{m}}$ when we take $\hat{p}_{\mathrm{I}}=\hat{p}_{\mathrm{ph}}$. Our preceding analysis reveals that $(\beta / \alpha)_{\mathrm{m}}\left(\hat{p}_{\mathrm{I}}\right)$ is exactly equal to $(\beta / \alpha)_{\mathrm{cr}}\left(\hat{p}_{\mathrm{I}}\right)$. This leads to the following important conclusion from our stability analysis:

If the healthy state $\mathcal{G}_{H}$ can be reached, then it is stable against dynamic remodeling.

As a consequence, two cases can occur:

- $\beta / \alpha<(\beta / \alpha)_{\mathrm{m}}\left(\hat{p}_{\mathrm{ph}}\right)$ : the homeostatic equilibrium state $\left(\lambda_{c}=\lambda_{c}^{\text {hom }}\right)$ for $\hat{p}=\hat{p}_{\mathrm{ph}}$ does not exist;

- $\beta / \alpha>(\beta / \alpha)_{\mathrm{m}}\left(\hat{p}_{\mathrm{ph}}\right)$ : the homeostatic equilibrium state $\left(\lambda_{c}=\lambda_{c}^{\text {hom }}\right)$ for $\hat{p}=\hat{p}_{\mathrm{ph}}$ exists, and is stable against remodeling. 
4.3 Stability analysis in case of a thick-walled artery

The thick-walled artery can be treated in an analogous way. In fact, we merely have to replace (40) by (36). However, when applying the method leading to Statement (57) we must realize that the perturbed variables $\lambda$, $\lambda_{\text {rec }}$, etc. are now all functions of the radial coordinate $R$ and time.

To find the function $L$, we have to integrate over the wall thickness of the artery. This is a cumbersome operation and therefore we prefer to use the second approach of Sect. 4.2. We assume (without an explicit proof as it seems quit logical, since the thin-walled artery is a limiting case of the thick-walled one) that again $(\beta / \alpha)_{\mathrm{cr}}=(\beta / \alpha)_{\mathrm{m}}$, and that when a healthy state can be reached, this state is stable.

Following the second approach of Sect. 4.2, we start from (36) and we denote by $P(k, \beta / \alpha)$ the right-hand side (36). Let the maximum $P_{\max }$ of $P$ be obtained for $k=k_{\mathrm{m}}$, then $k_{\mathrm{m}}=k_{\mathrm{m}}(\beta / \alpha)$ follows from

$\frac{\mathrm{d} P}{\mathrm{~d} k}\left(k_{\mathrm{m}} ; \frac{\beta}{\alpha}\right)=0$,

and

$P_{\max }=P_{\max }(\beta / \alpha)=P\left(k_{\mathrm{m}}(\beta / \alpha), \beta / \alpha\right)$.

The value of $(\beta / \alpha)_{\mathrm{m}}\left(p_{\mathrm{ph}}\right)$ is then defined as that value of $\beta / \alpha$ for which $P_{\max }$ is exactly equal to $p_{\mathrm{ph}}$. This means that for any $p_{\mathrm{I}}<p_{\mathrm{ph}},(\beta / \alpha)_{\mathrm{m}}\left(p_{\mathrm{I}}\right)$ follows from

$P_{\max }\left((\beta / \alpha)_{\mathrm{m}}\left(p_{\mathrm{I}}\right)\right)=p_{\mathrm{I}}$,

and on basis of its monotony (see Fig. 3) $(\beta / \alpha)_{\mathrm{m}}\left(p_{\mathrm{I}}\right)<(\beta / \alpha)_{\mathrm{m}}\left(p_{\mathrm{ph}}\right)$. We conclude thus that for all $\beta / \alpha>$ $(\beta / \alpha)_{\mathrm{m}}\left(p_{\mathrm{ph}}\right)$ all homeostatic equilibrium states for $p_{\mathrm{I}} \leq p_{\mathrm{ph}}$ exist and are stable against remodeling.

In the next section, we will present some explicit numerical results, which illustrate the procedures derived in this section.

\section{Results}

In this section, we show numerical results of the stability analysis presented in the previous section. We consider three cases to which we refer as: thin, cerebral and aorta; see Table 1. In order to examine whether the thin-walled approach is justified, we determine the condition of existence of the healthy state of a thin-walled artery with ratio of wall thickness to inner radius equal to 0.01 , using both the thin-walled and the thick-walled approach, and show that the results are the same. Then we determine the stability of a representative cerebral artery. Using the thinwalled approach we calculate $(\beta / \alpha)_{\text {cr }}$ according to stability Statement (57) as well as $(\beta / \alpha)_{\mathrm{m}}$ using the condition of existence of the healthy state (60). We show that explicit numerical values of $(\beta / \alpha)_{\mathrm{cr}}$ and $(\beta / \alpha)_{\mathrm{m}}$ are equal, thus confirming the conclusion of equivalence of stability condition and the existence of the healthy state. Finally, we study the stability of the aorta by evaluating the condition of existence of the healthy state using the thick-walled approach.

\subsection{Parameter settings}

For the demonstrative case of the thin artery we took the parameters of a cerebral artery, changing the ratio of wall thickness to radius only. The value of the homeostatic stretch $\lambda_{c}^{\text {hom }}$ varies from 1.012 to 1.2 in [15-19]. We arbitrarily set the value of the homeostatic stretch to 1.15 . The angles between the circumference and collagen fiber directions are taken from [16] for the media layer. We assume that the intrinsic properties of the matrix and collagen fibers do not differ much in the abdominal and cerebral arteries. Therefore, the shear modulus $c_{m}$ and material coefficients for collagen fibers $k_{1}$ and $k_{2}$ are obtained using the material parameters for elastin, ground substance and collagen for abdominal aorta in [16]. For that the relations (17) were used. The value of the initial recruitment 
Table 1 Model parameters of three types of arteries. The symbol '-' denotes the corresponding value for the aorta

\begin{tabular}{|c|c|c|c|c|c|}
\hline \multirow[t]{2}{*}{ Parameter } & \multicolumn{3}{|l|}{ Value } & \multirow[t]{2}{*}{ Unit } & \multirow[t]{2}{*}{ Description } \\
\hline & Aorta & Cerebral & Thin & & \\
\hline$H / R_{i}$ & 0.3 & 0.14 & 0.01 & {$[-]$} & $\begin{array}{l}\text { Ratio between the inner radius and the wall thickness } \\
\text { for unloaded artery at the initial state }\end{array}$ \\
\hline$\lambda_{c}^{\text {hom }}$ & 1.1 & 1.15 & 1.15 & {$[-]$} & Homeostatic collagen stretch \\
\hline$\gamma$ & $\pm \pi / 6$ & - & - & [rad] & Angles between the fibers and the circumferential direction \\
\hline$c_{m}$ & 75 & - & - & {$[\mathrm{kPa}]$} & Shear modulus of the matrix \\
\hline$k_{1}$ & 13 & - & - & {$[\mathrm{kPa}]$} & Stiffness parameter of collagen fiber \\
\hline$k_{2}$ & 10 & - & - & {$[-]$} & Material parameter of collagen fiber \\
\hline$\lambda_{\text {rec }, 0}$ & 1.0 & - & - & {$[-]$} & Initial recruitment stretch \\
\hline$\lambda_{z}$ & 1.3 & - & - & {$[-]$} & In-situ axial stretch of the artery \\
\hline$p_{\mathrm{ph}}$ & 16 & 10 & 10 & {$[\mathrm{kPa}]$} & Mean physiological blood pressure \\
\hline
\end{tabular}

Table 2 Values of $(\beta / \alpha)_{\mathrm{m}}$ for different pressures $p_{\text {I }}$ for the thin artery with $H / R_{i}=0.01$

\begin{tabular}{llllll}
\hline Pressure $p_{\mathrm{I}}(\mathrm{kPa})$ & & 1.6 & 4.4 & 7.2 & 10.0 \\
\hline$(\beta / \alpha)_{\mathrm{m}},(-)$ & Thin-walled approach & 14.2 & 55.1 & 96.0 & 136.8 \\
& Thick-walled approach & 14.3 & 55.4 & 96.5 & 137.5 \\
\hline
\end{tabular}

stretch $\lambda_{\text {rec, } 0}$ is arbitrarily set to one. In-situ axial stretch is taken from [22], and the blood pressure equals the mean intracranial cerebral blood pressure.

For a cerebral artery, the ratio of the wall thickness to the inner radius in the unloaded state is taken from [23].

For the aorta, the initial recruitment stretch $\lambda_{\text {rec }, 0}$ is arbitrarily set to 1.0 , and all the other parameters are obtained from the parameters in [16] (for the calculation of material parameters $c_{m}, k_{1}$ and $k_{2}$ the reader is referred to Sect. 3).

\subsection{Thin artery}

We calculated the minimal value $(\beta / \alpha)_{\mathrm{m}}$ for the thin artery of $H / R_{i}=0.01$, with the thin-walled approach using (60), and with the thick-walled approach, using (62) for four different values of the pressure $p_{\mathrm{I}}$; see Table 2 . The maximum in the set of the pressure values equals the mean intracranial cerebral blood pressure. The maximum relative error of the values of the ratio of $(\beta / \alpha)_{\mathrm{m}}$ obtained with the two models does not exceed $0.7 \%$, showing that the thin- and the thick-walled approaches are in good correspondence for really thin arteries.

\subsection{Cerebral artery}

For a representative cerebral artery with parameters given in Table 1 we determine $(\beta / \alpha)_{\text {cr }}$ as a function of applied pressure $\hat{p}_{\mathrm{I}}$ with the help of (58), and the expression $L(\lambda ; \beta / \alpha)=L_{\mathrm{cr}}$, which follows from (57). This is in accor-

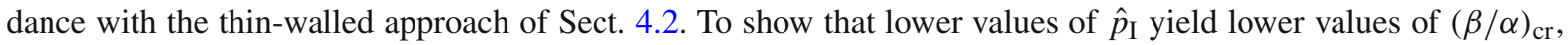
we plot $(\beta / \alpha)_{\text {cr }}$ as a function of the pressure; see the thick solid line in Fig. 3. As follows from (57), for $\left(\beta / \alpha, \hat{p}_{\mathrm{I}}\right)$ from the region above that line the state of the artery is stable, and for $\left(\beta / \alpha, \hat{p}_{\mathrm{I}}\right)$ from the region below that line the state is unstable. To find $(\beta / \alpha)_{\mathrm{m}}$ in accordance with the thick-walled approach of Sect. 4.3, we have to use (63). The thick dashed line in Fig. 3 represents the dependence of $(\beta / \alpha)_{\mathrm{m}}$ on $\hat{p}_{\mathrm{I}}$ obtained in this way. The graph shows that the values of $(\beta / \alpha)_{\mathrm{m}}$ obtained with the thin-walled approach are significantly lower than the corresponding values obtained with the thick-walled approach. The thin line on the graph displays the relation between $(\beta / \alpha)_{\mathrm{m}}$ and $p_{\mathrm{I}}$ in case of the absence of the matrix $\left(c_{m}=0\right)$, which is considered in Sect. 5.5. By comparing this case with 
the previous two, one can see that an order of magnitude larger values of $(\beta / \alpha)_{\mathrm{cr}}$ are needed to ensure stability in the case without matrix than in case with the matrix. We note that for the sake of getting comparable values of the pressure both in thin and thick-walled cases, we made the pressure dimensionless as defined by (42).

We now proceed with analyzing the expression (59) representing the function $\hat{P}$ of $\lambda$ with $\beta / \alpha$ as a parameter. For different values of $\beta / \alpha$, this function is presented in Fig. 4. The lines in the graph are bounded from above. Hence, the pressure of the homeostatic equilibrium states is bounded from above as well. The line for $\beta / \alpha=(\beta / \alpha)_{\mathrm{m}}\left(\hat{p}_{\mathrm{ph}}\right)$, reaches in its peak value the physiological pressure $\hat{p}_{\text {ph }}$. The value $(\beta / \alpha)_{\mathrm{m}}$ is the minimal value of the ratio $\beta / \alpha$ for which the healthy pressure can be reached. For $\beta / \alpha<(\beta / \alpha)_{\mathrm{m}}$, the pressure values do not reach the pressure $\hat{p}_{\text {ph }}$, which means that for this value of $\beta / \alpha$ the healthy state does not exist. On the other hand, the healthy state exists for any ratio satisfying the condition $\beta / \alpha>(\beta / \alpha)_{\mathrm{m}}$, and the corresponding line (the dash-dot line) reaches $\hat{p}_{\text {ph }}$ before reaching its peak value.

We note that in the considered thin-walled case $(\beta / \alpha)_{\mathrm{m}}=(\beta / \alpha)_{\mathrm{cr}}$, which is in line with the conclusion formulated at the end of Sect. 4.2. From the thin-walled approach we find $(\beta / \alpha)_{\mathrm{m}}=0.68$, whereas from the thick-walled approach we find $(\beta / \alpha)_{\mathrm{m}}=1.55$, which is more than twice as high.

\subsection{Aorta}

As a representative case of a thick-walled artery we consider the aorta. The parameters for the aorta are taken from [16]. Note that the physiological pressure here $(16 \mathrm{kPa})$ is higher than the one for the thinner cerebral artery $(10 \mathrm{kPa})$. Our calculations showed that the homeostatic state for the aorta is unconditionally stable (stable for $(\beta / \alpha)_{\mathrm{m}}=0$ already) according to the thin-walled approach, whereas $(\beta / \alpha)_{\mathrm{m}}=0.75$ according to the thick-walled approach.

\subsection{Effect of uniform matrix degradation on the stability of the artery}

In this section we analyse the stability in case of uniform matrix degradation. This is inspired by the observation that the content of elastin is decreased in aneurysms (e.g. [24,25]). We can model this by decreasing the shear modulus of the matrix. All results derived here remain valid also in the case of dynamic uniform degradation, provided the rate of degradation is slow compared to the rate of remodeling. Indeed, the time scale of degradation of elastin in aneurysmal tissues is in the order of years, while the half-life of collagen is 3-90 days in various soft tissues [26]. We notice that in case of non-uniform degradation the cylinder will no longer preserve the cylindrical shape, and then the stability analysis would be completely different. We assume, therefore, a uniform quasi-static degradation, and the only consequence is that we have to replace in (40) the shear modulus $c_{m}$ by a smaller value $c_{m}(t)$, where $c_{m}(t)$ is the degraded value of $c_{m}$ at the current time $t$ with respect to the original healthy value of $c_{m}$. The most critical situation is then obtained by letting $c_{m} \rightarrow 0$. Relation (42) then reduces to

$\frac{2 n_{c}(t)}{\lambda^{2}(t)+\lambda_{z}^{2} \tan ^{2} \gamma} \hat{\tau}_{f}\left(\lambda_{c}(t)\right)=\hat{p}_{\mathrm{I}}$,

where the pressure $p$ is scaled according to (42) with respect to the original value of the matrix shear modulus $c_{m}$ in order to keep the same dimensionless value of the pressure when degrading the matrix. The rest of the analysis is exactly analogous to that for the non-degraded case, ultimately resulting in the following expressions for $L$ and $\Pi_{I}$ (compare with (52)-(54) and (58))

$L=\frac{A}{C}$,

$A=-\frac{2 \hat{\tau}_{f}^{\text {hom }}}{\lambda^{2}+\lambda_{z}^{2} \tan ^{2} \gamma}\left[\frac{\beta}{\alpha}-\frac{2 n_{c} \tilde{\tau}_{f}^{\text {hom }}}{\lambda_{\text {rec }}}\right]$, 
$C=\frac{4 n_{c} \hat{\tau}_{f}^{\mathrm{hom}} \lambda}{\left(\lambda^{2}+\lambda_{z}^{2} \tan ^{2} \gamma\right)^{2}}\left(\tilde{\tau}_{f}^{\mathrm{hom}}-1\right)$,

and

$\Pi_{I}\left(\lambda ; \frac{\beta}{\alpha}\right) \equiv\left[\frac{\beta}{\alpha}\left(\frac{\Lambda(\lambda)}{\lambda_{c}^{\text {hom }}} \cos \gamma-\lambda_{\text {rec }, 0}\right)+n_{c, 0}\right] \frac{4 \hat{\tau}_{f}^{\text {hom }}}{\Lambda^{2}(\lambda)}=\hat{p}_{\mathrm{I}}$,

which allows determining the critical value of the ratio $(\beta / \alpha)_{\mathrm{cr}}^{\mathrm{col}}$ in case of a tissue consisting of collagen only. Each value of $\beta / \alpha$ satisfying $\beta / \alpha>(\beta / \alpha)_{\mathrm{cr}}^{\mathrm{col}}$ ensures stability of the homeostatic state of the tissue without matrix. An important interpretation of this result is the following: suppose the tissue is in a healthy homeostatic state; if now the matrix degrades completely, a new homeostasis will be reached and be stable against dynamic remodeling, provided that

$\beta / \alpha>(\beta / \alpha)_{\mathrm{cr}}^{\mathrm{col}}$.

\section{Discussion}

We have calculated the values $(\beta / \alpha)_{\mathrm{m}}$ for both an a priori thin-walled approximation and for the more general thick-walled model. We have verified the thin-walled approach by considering the thick-walled model for the case $H / R_{i}=0.01$. The error in $(\beta / \alpha)_{\mathrm{m}}$ was less than $0.7 \%$, confirming the correctness of the thin-walled approach in the limit of $H / R_{i} \rightarrow 0$.

We compared the values of $(\beta / \alpha)_{\mathrm{m}}$ obtained using the thick-walled approach with the corresponding values obtained using the thin-walled approach both for cerebral artery and for abdominal aorta. In case of a cerebral artery $\left(H / R_{i}=0.14\right)$ the relative error in $(\beta / \alpha)_{\mathrm{m}}$ was about $60 \%$. In case of the abdominal aorta the thin-wall approach yielded an unconditional stability, meaning that even for $\beta / \alpha=0$ the artery is stable, whereas the thick-walled model yielded $(\beta / \alpha)_{\mathrm{m}}=0.75$. We conclude that even in case of the (thinner) cerebral artery the thin-walled approach does not yield an accurate estimate of $(\beta / \alpha)_{\mathrm{m}}$.

Recalling the definition of the dimensionless pressure (42), one can see that under the same pressure and geometry of the artery, increasing the matrix shear modulus corresponds to decreasing the dimensionless pressure. On the other hand, Fig. 3 shows that the value of $(\beta / \alpha)_{\mathrm{m}}$ increases with an increase of the dimensionless pressure. From this we conclude that the stiffer the matrix (or the lower the dimensionless pressure on the graph), the lower value of $(\beta / \alpha)_{\mathrm{m}}$ is needed to provide stability of the healthy state.

Watton et al. [15] and [16] question whether a linear function of the remodeling equations (12) realistically represents the remodeling behavior of the tissue, suggesting that the nonlinear functional forms might be more appropriate. Towards this end, we note that our linear stability analysis is not restricted to linear remodeling equations. In case of nonlinear remodeling equations, still of the form (12), but with nonlinear functions of $\lambda_{c}-\lambda_{c}^{\text {hom }}$ at their right-hand sides, the rate constants $\alpha$ and $\beta$ should be treated as the coefficients of the first-order terms of a Taylor expansion of these nonlinear functions around $\lambda_{c}=\lambda_{c}^{\text {hom }}$.

\section{Conclusions}

We achieved the goal of this paper to study stability of the homeostatic state of an artery against remodeling as described by a model of remodeling of an arterial tissue presented by Watton et al. [15]. In this model the remodeling is described by the evolution of collagen recruitment stretch and collagen density. In the equilibrium state all collagen fibers are uniformly stretched to the homeostatic stretch $\lambda_{c}^{\text {hom }}$. If the artery is out of equilibrium, collagen recruitment stretch and collagen density are described by evolution equations with rate parameters $\alpha$ and $\beta$, respectively. 
The presented stability analysis is linear. To investigate the stability we disturb the healthy artery (stationary state) by small dynamic perturbations. Whether, via dynamic remodeling, the artery will recover equilibrium depends on the ratio $\beta / \alpha$ of the rate constants of the remodeling variables. Use of the assumption that the equilibrium state of a healthy artery is stable against remodeling it enabled us to determine the range of physiologically acceptable values of the rate constants.

We have considered as a specific example a thin-walled tube under internal pressure and axial pre-stretch. The tube represents a healthy artery, and the pressure and the pre-stretch describe physiological loading. We showed that the artery is stable against the remodeling if $\beta / \alpha>(\beta / \alpha)_{\mathrm{m}}$, where the value of $(\beta / \alpha)_{\mathrm{m}}$ is determined by $L(\lambda ; \beta / \alpha)=L_{\mathrm{cr}}$ and $\Pi_{I}(\lambda ; \beta / \alpha)=\hat{p}_{\mathrm{I}}$ (see (57) and (58) for the definitions). One of our main results is that the conditions for existence of the healthy state and for stability of this state are equivalent. This theoretical finding was confirmed by the numerical results in case of a thin-walled approach. The equivalence of the two methods holds also in the more general case of a thick-walled artery, which enabled us to study the stability of the thick-walled artery by considering the existence of the healthy state only.

Comparison of the thin-walled and the thick-walled approach for the relatively thin-walled cerebral artery yielded already large difference in $(\beta / \alpha)_{\mathrm{m}}$ between these two approaches.

Reduction of the matrix stiffness, considered a trigger for aneurysm formation, yielded an increase of $(\beta / \alpha)_{\mathrm{m}}$, with a finite value of $(\beta / \alpha)_{\mathrm{m}}$ for complete absence of the matrix. This implies that if $\beta / \alpha>(\beta / \alpha)_{\mathrm{m}}$ then after any, partial or total, matrix degradation the artery will remodel into a stable healthy state.

In this paper we determined physiologically acceptable ranges for the ratio of the two rate constants in the relatively simple remodeling model of Watton and Hill. We recommend analogous studies for recent, more complex models of remodeling of the vascular wall.

Acknowledgement This project is supported by a grant from Philips Medical Systems, which is gratefully acknowledged.

Open Access This article is distributed under the terms of the Creative Commons Attribution Noncommercial License which permits any noncommercial use, distribution, and reproduction in any medium, provided the original author(s) and source are credited.

\section{References}

1. Humphrey JD (2001) Stress, strain, and mechanotransduction in cells. J Biomech Eng 123:638-641

2. Fung YC (1990) Biomechanics: motion, flow, stress, and growth. Springer, New York

3. Humphrey JD, Canham PB (2000) Structure, mechanical properties, and mechanics of intracranial saccular aneurysms. J Elast 61:49-81

4. Skalak R, Dasgupta G, Moss M, Otten E, Dullumeijer P, Vilmann H (1982) Analytical description of growth. J Theor Biol 94:555577

5. Rodriguez EK, Hoger A, McCulloch AD (1994) Stress-dependent finite growth in soft elastic tissues. J Biomech 4:455-467

6. Taber LA, Eggers DW (1996) Theoretical study of stress-modulated growth in the aorta. J Theor Biol 4:343-357

7. Taber LA, Humphrey JD (2001) Stress-modulated growth, residual stress, and vascular heterogeneity. J Biomech Eng 6:528-535

8. Kuhl E, Maas R, Himpel G, Menzel A (2007) Computational modeling of arterial wall growth. Biomech Model Mechanobiol 6:321-331

9. Rachev A, Stergiopulos N, Meister JJ (1996) Theoretical study of dynamics of arterial wall remodeling in response to changes in blood pressure. J Biomech 29:635-642

10. Driessen NJB, Wilson W, Bouten CVC, Baaijens FPT (2004) A computational model for collagen fibre remodelling in the arterial wall. J Theor Biol 226:53-64

11. Driessen NJB, Bouten CVC, Baaijens FPT (2005) A structural constitutive model for collagenous cardiovascular tissues incorporating the angular fiber distribution. J Biomech Eng 3:494-503

12. Driessen NJB, Cox MAJ, Bouten CVC, Baaijens FPT (2008) Remodeling of the angular collagen fiber distribution in cardiovascular tissues. Biomech Model Mechanobiol 2:93-103

13. Gleason RL, Taber LA, Humphrey JD (2004) A 2-D model of flow-induced alterations in the geometry, structure, and properties of carotid arteries. J Biomech Eng 3:371-381

14. Gleason RL, Humphrey JD (2005) A 2D constrained mixture model for arterial adaptations to large changes in flow, pressure and axial stretch. Math Med Biol 4:347-369

15. Watton PN, Hill NA, Heil M (2004) A mathematical model for the growth of the abdominal aortic aneurysm. Biomech Model Mechanobiol 3:98-113 
16. Watton PN, Hill NA (2009) Evolving mechanical properties of a model of abdominal aortic aneurysm. Biomech Model Mechanobiol 8:25-42

17. Baek SR, Rajagopal KR, Humphrey JD (2005) Competition between radial expansion and thickening in the enlargement of an intracranial saccular aneurysm. J Elast 80:13-31

18. Baek SR, Rajagopal KR, Humphrey JD (2006) A theoretical model of enlarging intracranial fusiform aneurysms. J Biomech Eng 128:142-149

19. Kroon M, Holzapfel GA (2007) A model for saccular cerebral aneurysm growth by collagen fibre remodelling. J Theor Biol 247:775-787

20. Watton PN, Ventikos Y, Holzapfel GA (2009) Modelling the growth and stabilization of cerebral aneurysms. Math Med Biol. doi:10.1093/imammb/dqp001

21. Watton P, Ventikos Y (2009) Modelling evolution of saccular cerebral aneurysms. J Strain Anal 44:375-389

22. Monson KL, Goldsmith W, Barbaro NM, Manley GT (2003) Axial mechanical properties of fresh human cerebral blood vessels. J Biomech Eng 125:288-294

23. Walmsley JG, Campling MR, Chertkow HM (1983) Interrelationships among wall structure, smooth muscle orientation, and contraction in human major cerebral arteries. Stroke 14:781-790

24. He CM, Roach MR (1994) The composition and mechanical properties of abdominal aortic aneurysms. J Vasc Surg 20:6-13

25. Carmo M, Colombo L, Bruno A, Corsi FR, Roncoroni L, Cuttin MS, Radice F, Mussini E, Settembrini PG (2002) Alteration of elastin, collagen and their cross-links in abdominal aortic aneurysms. Eur J Vasc Endovasc Surg 23:543-549

26. Humphrey JD (1999) Remodeling of a collagenous tissue at fixed lengths. J Biomech Eng 121:591-597 